\title{
28 Research Square \\ Suppressing COVID-19 transmission in Hong Kong: an observational study of the first four months
}

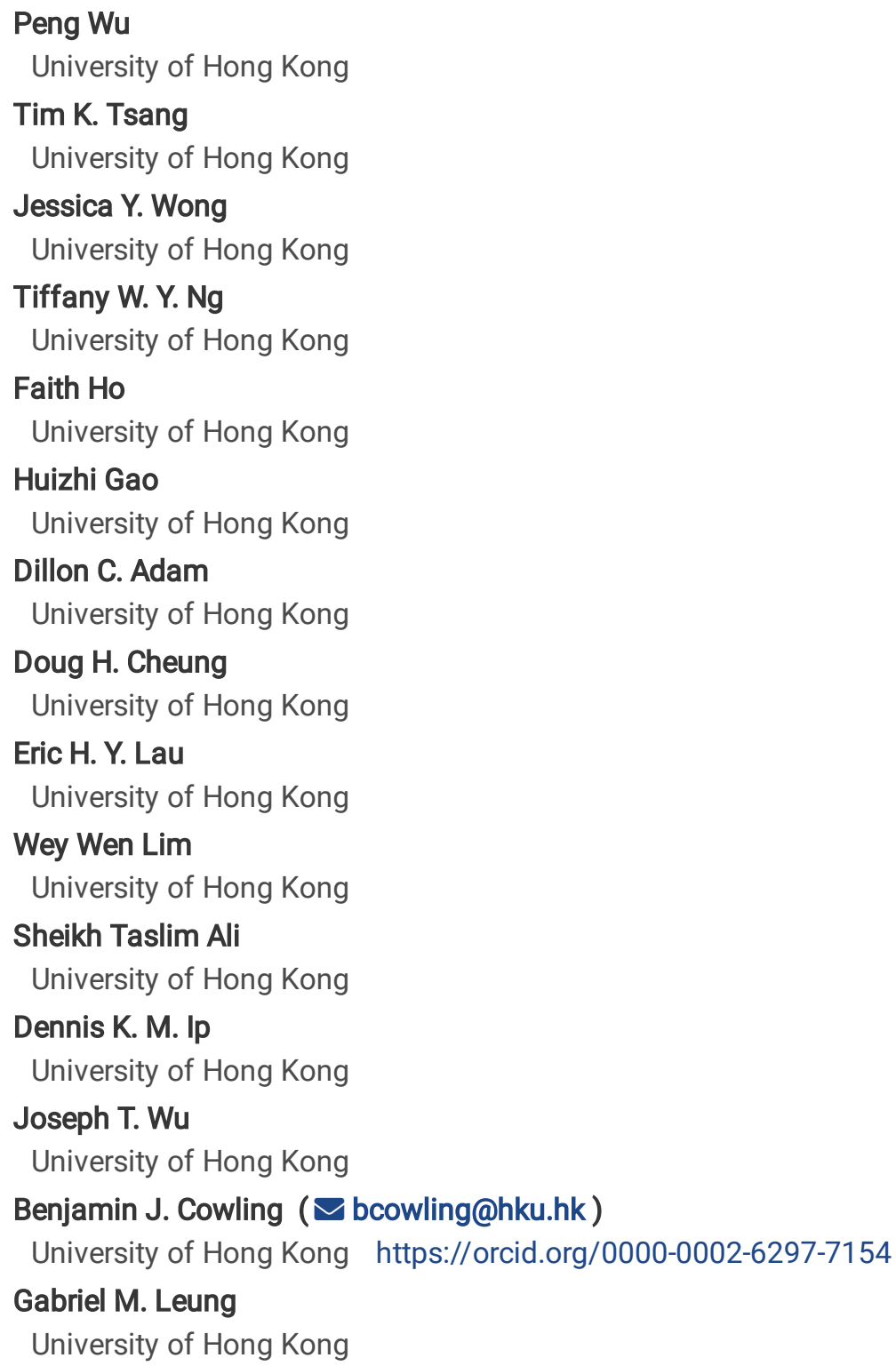




\section{Abstract}

Background: Hong Kong was one of the first locations outside of mainland China to identify COVID-19 cases in January 2020. We assessed the impact of various public health measures on transmission.

Methods: We analysed data on all COVID-19 cases and public health measures in Hong Kong up to 7 May 2020. We described case-based, travel-based and community-based measures and examined their potential effects on case identification and transmission. Changes in transmissibility measured by the effective reproductive number $R_{t}$ were estimated by comparing the $R_{t}$ between periods when public health measures were and were not in effect. Delays in case confirmation in imported cases and locally infected cases were analysed to indicate the possible impact of expansion of laboratory testing capacity.

Findings: Introduction of a 14-day quarantine on persons arriving from affected areas was associated with a $95 \%$ reduction in transmissibility from imported cases. Testing all arriving travelers reduced mean delays between arrival and detection of imported cases. Increases in laboratory testing capacity for pneumonia inpatients and symptomatic outpatients reduced the delay from onset to confirmation. Working from home and physical distancing measures implemented in high-risk facilities were associated with $67 \%$ and $58 \%$ reductions in transmission of COVID-19, respectively.

Interpretation: Suppression of COVID-19 transmission in the first pandemic wave in Hong Kong was achieved through integration of travel-based, case-based and community-based public health measures focusing on early case identification and isolation and physical distancing.

\section{Introduction}

Coronavirus disease COVID-19, caused by the Severe Acute Respiratory Syndrome coronavirus SARS-CoV-2, rapidly spread around the world after it was first identified in Wuhan in December 2019 1-3. As of 7 May 2020, over 3.7 million COVID-19 cases have been reported globally in more than 200 jurisdictions ${ }^{4}$. Intense control measures such as complete lockdowns and travel restrictions have been implemented in locations with large numbers of COVID-19 cases $^{5}$.

Hong Kong was one of the first locations outside of mainland China to identify COVID-19 cases, and acted swiftly to implement a series of public health measures ${ }^{6}$. As of May 7, Hong Kong has reported 1045 COVID-19 cases and 4 deaths, which are 143 cases and 0.5 deaths per million population, respectively, but has not yet experienced a locally sustained outbreak ${ }^{7}$. Here, we describe the public health measures taken in Hong Kong and estimate the relative contributions of case-based measures, community-based measures and travel-based measures to suppress COVID-19 transmission.

\section{Methods}

\section{Sources of data}

Detailed demographic, clinical and epidemiological data on all probable and laboratory-confirmed cases were compiled into an electronic "eCOVID-19" database. COVID-19 has been a notifiable disease in Hong Kong since 8 January 2020. Although some SARS-CoV-2 infections are asymptomatic, 8,9 for simplicity we use the term "COVID-19 case" here to include asymptomatic as well as symptomatic SARS-CoV-2 infections. Patients who meet certain clinical, epidemiologic and/or laboratory criteria would be classified as suspected, probable or confirmed cases in Hong Kong (Appendix). A patient who has symptom(s) indicating an acute respiratory infection and reports a COVID-19 related epidemiologic link would be a suspected COVID-19 case and should be reported to the Department of Health. A probable case is a suspected case having more specific clinical and epidemiologic evidence for COVID-19 but without a conclusive laboratory test result for infection with SARS-CoV-2. A confirmed case is a patient having specimens tested indicating virologic or serologic evidence of infection with SARS-CoV-2, irrespective of symptoms or epidemiologic linkage of the case.

After confirmation of a COVID-19 case, an investigation is carried out to identify other connected cases and possible chains of transmission. A close contact of a COVID-19 case is a person who stayed in the same confined area without face-to-face interactions with a confirmed COVID-19 case for more than two hours when both of them were not wearing face masks, or if they 
had a face-to-face interaction for more than 15 minutes when the COVID-19 case was not wearing a face mask. Non-close contacts who had brief contact with the case but not satisfying the definition of close contacts are defined as other contacts. The earliest confirmed COVID-19 case (index case) who triggered the contact tracing investigation might not be the one initiating the transmission of the cluster if earlier infected cases were subsequently detected, i.e. the index case might not be the primary case in a cluster ${ }^{10}$.

The government has repurposed several public facilities to quarantine asymptomatic people who are either close contacts of confirmed COVID-19 cases or travellers from areas with a high-risk transmission of COVID-19, or who violated the order of mandatory home quarantine issued by the Department of Health, or who may not be able to arrange home quarantine after returning Hong Kong from overseas. All asymptomatic close contacts of COVID-19 cases underwent medical observation at the quarantine centres for 14 days and medical surveillance for another 14 days, and a RT-PCR test during quarantine or after completion of quarantine, or sent to hospital for testing and management when acute respiratory symptoms were reported. Other contacts will be put under medical surveillance for 28 days and they will be sent to hospital for testing and management when acute respiratory symptoms were reported.

Information on interventions applied in Hong Kong in response to COVID-19 was collected and classified as travel-based, casebased and community-based measures. Travel-based measures include travel restrictions, port control and inbound traveler screening. Case-based interventions refer to measures related to case identification and isolation, and quarantine of contacts of confirmed cases. Community-based measures included physical distancing measures and behavioral changes in the population as a whole. A summary of daily numbers of persons tested and confirmed at various settings was obtained from the Department of Health.

We conducted five cross-sectional telephone surveys in adults living in Hong Kong, on 20-23 January, 11-14 February, 10-13 March, 31 March - 3 April and 5-8 May. These surveys used similar instruments to the surveys that we carried out in Hong Kong during the SARS epidemic in $2003^{11,12}$ and the influenza $A(H 1 N 1)$ pdm09 pandemic in $2009^{13}$. Participants provided informed consent and were asked about their risk perception and their behaviours related to physical distancing and personal hygiene.

\section{Statistical analysis}

We described the demographic and epidemiologic characteristics of all COVID-19 cases identified in Hong Kong. The epidemic curves were constructed by stratifying cases into 6 groups, specifically, "sporadic imported cases", "imported index cases", "linkedimported cases", "sporadic local cases", "index local cases" and "linked-local cases" (Appendix), and analysed by date of illness onset for symptomatic infections, and by date of confirmation for cases that were detected while asymptomatic. We analysed the case detection rate with the data collected through the infectious disease notification system and the enhanced laboratory surveillance programme by setting, such as hospital, outpatient clinic, temporary test centre etc., where each case was identified and the numbers of tests done in each setting.

We analysed the delays between illness onset and case report in linked local cases as an indicator of the potential impact on transmissibility of isolation of cases. Among quarantined contacts, we analysed the reported number of contacts traced and the confirmation delays from symptom onset for symptomatic cases and from last exposure for asymptomatic cases, and examined the average number of contacts traced and the occurrence of cases to explore the potential impact on transmissibility of contact tracing and quarantine. We examined the distributions of these metrics over time.

We extended the approach described by Cori et $\mathrm{al}^{14}$. to estimate the effective reproductive number for imported and local cases separately in the context of different types of interventions because travel-based measures would directly impact on infections identified from inbound travelers through medical observation or quarantine while community-based measures were assumed to affect occurrence of local infections ${ }^{15}$. We first used the deconvolution approach in Goldstein et al. ${ }^{16}$, with estimated incubation period with log-normal distribution with mean 5.2 days (SD 3.9) from Li et al. ${ }^{2}$. to obtain the epidemic curve by infection date (Appendix). Then we estimated the time-varying reproductive number for imported and local cases from infectiousness since infection, generated by incubation period distribution and the infectiousness relative to onset time ${ }^{17}$ (Appendix) to account for presymptomatic infectiousness, and incidence of COVID-19 cases over time. We assumed the imported cases started to contribute their infectiousness at their onset since they spend their incubation period outside Hong Kong (Appendix). Since the source of 
infection for sporadic local cases or local index cases were missing (could be from imported cases or other local cases), we developed a data-augmented Markov chain Monte Carlo algorithm to jointly estimate the time-varying reproductive numbers for local and imported cases, and the source of infection for local cases (Appendix). Time-varying estimates of effective reproductive numbers were made with a 7-day sliding window. We used Gamma $(1,5)$ as a prior distribution for $R_{t}$, with mean and standard deviation equal to 5 . This is an uninformative and conservative prior for the $R_{t}$ so that when the posterior estimate of $R_{t}$ is below one, the epidemic is under control ${ }^{14}$.

To estimate the effect of interventions on the time varying reproductive number, we refitted the above models, assuming that $R_{t}$ was log-linearly associated with the implementations of interventions and estimated the effectiveness of interventions, defined as one minus change in $R_{t}$ before and after implementations of interventions (Appendix). We jointly estimated the effectiveness of the three interventions in the model: 1) mandatory quarantine of inbound travelers, 2) civil servants working from home (that was widely mirrored in other sectors of the economy), 3) additional physical distancing measures including closure of high-risk places/facilities. To account for the uncertainty of the sampling of incubation period for each individual, we used the bootstrap approach in Salje et al. ${ }^{18}$, to derive the $95 \%$ credible intervals $\left(95 \% \mathrm{Crl}\right.$ ) for the estimate of $R_{t}$ and the effectiveness of target interventions (Appendix). Survey data were weighted by age and sex to the local adult population. All analyses were conducted in $\mathrm{R}$ version 4.0.0 (R Foundation for Statistical Computing, Vienna, Austria).

\section{Ethical approval}

Our project was approved by the Institutional Review Board of the University of Hong Kong.

\section{Results}

As of 7 May, there have been 1,045 COVID-19 cases identified in Hong Kong, including 1044 confirmed cases and 1 probable case. Of these, 680 (65\%) were sporadic imported cases, and the remainder includes $32(3 \%)$ imported index cases in clusters, 67 (6\%) local cases linked to imported cases, 68 (7\%) sporadic local cases or index cases in clusters, and $198(19 \%)$ linked local cases (Table 1). The only probable case was a returnee from the Diamond Princess Cruise. The majority of the confirmed cases were young and middle-age adults while the local cases were generally older and more likely to report underlying medical conditions than the imported cases (Table 1). While most infections were mild, a total of 64/1045 (6.1\%) of cases have had documented measurement of oxygen saturation $<95 \%$. This fraction was higher among local sporadic and index cases (Table 1).

From the start of the epidemic, all suspected and confirmed COVID-19 cases were isolated in designated public hospitals. Testing initially focused on cases with relevant clinical presentations and travel history to affected areas, and was expanded from the end of January 2020 to all inpatients with pneumonia regardless of travel history, and then expanded to all outpatients with relevant clinical presentations in February. Given increases in the numbers of cases among arriving travellers particularly in March, from 25 March onwards, non-Hong Kong residents have been barred from entry, and all flight transit services have been suspended in Hong Kong.

Since late January, a series of intense community-based measures had been applied, including closure of all local schools and theme parks and suspension of social events after the Lunar New Year, special arrangements for civil servants to work from home until early May with a short period of suspension of this measure in March, and closure of high-risk facilities including bars, nightclubs, fitness centres and theatres, and restrictions in population mixing in designated places such as restaurants and other public areas from the end of March (Appendix).

\section{Impact of travel measures}

Travel reductions and restrictions had a considerable impact on the numbers of persons arriving in Hong Kong. Since early February 2020, 12 out of 14 land border control points in Hong Kong have been temporarily suspended. Non-Hong Kong residents have been barred from entering Hong Kong from 25 March leading to a substantial reduction in passengers through the airport, from an average of around 200,000 arrivals per day in April 2019 down to an average of 500 per day in April 2020. 
Figure 1A shows the incidence of infections among imported cases and the small number of cases identified among their close contacts. The locations of imported cases changed over time, and the largest number of imported infections were identified in travellers arriving from the United Kingdom and other European countries (Figure S1). Notably there was a considerable increase in the proportion of asymptomatic infections identified after 24 March when testing was expanded to all inbound travellers regardless of symptoms, from $11 \%(31 / 286)$ to $37 \%$ (119/320). Some asymptomatic COVID-19 cases would have gone on to develop symptoms later but this was not recorded in our dataset. Based on the small numbers of local infections linked to the imported cases, we estimated the effective reproductive number from imported cases was maintained well below 1 since mid-February particularly after early March (Figure 1B). We estimated that the effectiveness of 14-day mandatory quarantine for travellers since February 8 was $95 \%$ (95\% Crl : $88,98 \%)$.

Most of the imported cases were detected either through testing patients meeting the reported criteria or the screening tests on symptomatic or asymptomatic inbound travellers. A substantial increase was observed in patients who met the reporting criteria and were tested since mid-March, and the proportion of confirmed cases among the tested has also doubled since early March.

\section{Impact of community-based measures}

Figure 1C shows the epidemic of local infections, including local sporadic and index cases, and the linked cases from clusters. Assuming that all transmission chains must ultimately lead back to the original epicentre of Wuhan, it is likely that there were missed connections or missed cases occurring between the local cases and some of the imported cases or their contacts. Estimates of the effective reproductive number for local infections are shown in Figure 1D. One of the most consequential physical distancing measures appears to be the work at home policy for civil servants, which was mirrored by many other institutions and private employers in February, but was lifted for the first three weeks in March. Coincidentally, transmissibility rose after this measure was lifted (Figure 1D), and this surge in local infections in March was associated with a number of clusters associated with gathering in restaurants and bars, the largest of which was connected to a band that played in a number of bars across Hong Kong and the customers of those bars. As a result, the government instituted new requirements for restaurants in late March and closed all bars on 3 April (Appendix). These measures together with the reinstitution of working from home from 21 March were associated with a reduction in transmissibility back down below 1 . We estimated that the effectiveness of implementation of civil servants working from home was $67 \%(95 \% \mathrm{Crl}: 36 \%, 90 \%)$ and the effectiveness of implementation of additional physical distancing measure including closure of high-risk places/facilities on March 28 was 58\% (95\% Crl: 15\%, 99\%).

\section{Impact of case-based measures}

Laboratory testing capacity had been gradually expanded over time (Figure 2), from around 600 tests per day in early February to 2000-4000 tests per day in late March until May. The median time delays from symptom onset to isolation of the local sporadic cases and index cases gradually reduced over time from 10 days in late January and early February to 5 days in late March and early April (Figure $3 \mathrm{~A}$ ) but more than $59 \%$ of local cases in March were isolated $\geq 5$ days after onset. The time delays were relatively shorter in identifying linked local cases (Figure 3B), with a median of 9.5 days and 4 days in the two time periods respectively.

As of 7 May 2020, more than 170,000 specimens had been tested in Hong Kong. Most of the specimens tested were collected from pneumonia inpatients and inbound travellers. The overall detection proportions of COVID-19 cases were the highest in specimens collected from suspected cases and symptomatic travellers returning from overseas (Figure 2B-F), with more than half of the cases in Hong Kong identified from these individuals.

As of 7 May, in total 5265 contacts including 2840 close contacts of confirmed cases had been sequestered in quarantine centres in Hong Kong, and 99 cases were identified among these quarantined persons. There were around 12 "other contacts" for every confirmed case placed under medical surveillance based on the data collected by $20 \mathrm{March}$, and no cases were identified in these persons. A slightly declining trend was observed in the number of close contacts of imported and local cases traced during the first wave (Figure 3E). There seemed no visible correlation between the number of contacts traced and the delay in confirmation of infection (Figure 3F).

\section{Impact of behavioral changes}


A number of notable behavioral changes occurred over time in the local community, measured by our serial surveys (Figure 4). Respondents to the surveys reported greater personal hygiene from late January onwards, and the use of face masks in public exceeded $98 \%$ by mid-February. However, it should be noted that face mask usage was high even when the local effective reproductive number exceeded 1 in March (Figure 1D, Figure S2-S5). In our survey on 10-13 March, 506 respondents reporting being employed in February, and 298/506 (59\%) of these respondents reported changes in their hours at workplace, with 177/506 (35\%) working at home for $\geq 2$ days per week.

\section{Discussion}

Hong Kong managed to suppress the first epidemic wave of COVID-19. While there were undoubtedly more than 1045 infections with SARS-CoV-2, testing of all inpatients with pneumonia gives reassurance that a large epidemic could not have occurred unnoticed. A previous study estimated that the risk of hospitalization increased from about $1 \%$ in infected $20-29$ year olds to $18 \%$ in infected persons $\geq 80$ years of age ${ }^{19}$. Increases in detections of asymptomatic cases among travellers from late March onwards (Figure 1A) once testing was expanded to cover asymptomatic arriving persons suggest that earlier asymptomatic COVID-19 cases might have been missed. By 7 May 2020 there were 4 recorded COVID-19 deaths in Hong Kong among the 1045 cases (0.4\%). This low mortality rate among cases is likely due to a relatively small number of older adults among the cases (Table 1), and aggressive treatment 20 .

Our findings show that moderate physical distancing measures were a critical component in successful suppression of the first wave. Test-and-trace were used throughout the first wave but were insufficient to prevent $R_{t}$ rising above 1 in March. When the government implemented work-from-home for civil servants in February, many local organizations and businesses followed suit, with more than half of working adults in Hong Kong reducing the hours spent at their workplace. When this policy was relaxed in early March it coincided with a surge in local transmission that only declined below 1 in late March after the work-from-home policy was reactivated (Figure 1D). Although this is circumstantial evidence, and an ecological association may not be causal, it is plausible that physical distancing measures such as working from home could play a major role in reducing transmission.

Isolation of all suspected and confirmed cases in hospital has likely contributed to reducing onwards transmission, although many cases have been confirmed more than a week after illness onset (Figure 3). Expansion in testing to outpatients had a clear effect in reducing onset-to-confirmation delays (Figure 3). Infectiousness is likely to be greatest around the time of illness onset ${ }^{17}$, and shortening of onset-to-confirmation delays should be a continued priority.

Tracing of close contacts had also likely contributed to reducing community transmission. However, the number of contacts traced for each case (Figure 3E) was much lower than the average of 27 contacts per case in Taiwan ${ }^{21}$. This might be related to the wide use of surgical mask in the general public in Hong Kong since January. Among the 212 cases identified through contact tracing, only 92 of these were in persons quarantined in designated facilities while the remaining were persons found to have symptoms at contact tracing and sent to hospitals directly by the Department of Health. In a more detailed investigation of clustering of infections and super-spreading events, we estimated that approximately $20 \%$ of cases were responsible for $80 \%$ of COVID- 19 transmission in Hong Kong ${ }^{22}$. In that study we also examined chains of transmission and found that only one third of terminal nodes, i.e. the cases at the end of transmission chains, occurred in quarantined persons.

It is likely that widespread use of face masks in the community have played a role in reducing transmission ${ }^{23-26}$. Notably, the largest clusters in Hong Kong were associated with transmission in bars and at a wedding dinner ${ }^{22}$, both locations in which face masks were not worn. However, our findings indicate that universal community masking in March was insufficient to prevent the effective reproductive number from rising above 1 (Figure 1D).

Although each of the interventions discussed above may not have been sufficient to prevent a large epidemic of COVID-19 if deployed alone, the use of these measures in combination has been able to successfully prevent an epidemic. One implication of our findings is that if some interventions had not been in place, for example if test-and-trace had not been implemented locally, it probably would have been necessary to increase the intensity of some of the other measures such as physical distancing. As a corollary, in considering lifting of these measures, they should be done in deliberate phases and in a staggered manner so as to be able to identify which relaxed intervention(s) may subsequently lead to recrudescence. 
There are a number of limitations of our study. First, we reclassified the COVID-19 cases identified in Hong Kong into six groups based on the information collected in epidemiologic investigations particularly taking into account different approaches used in case identification and their potential impact on local transmission of COVID-19. Limited information might lead to misclassifications of cases especially for those in older age, being identified late after symptom onset or having severe infections. Second, we applied statistical approaches to investigate the transmission of COVID-19 and estimate the effectiveness of public health interventions. A mechanistic model may be better to use in the future to demonstrate the underlying mechanisms of interventions considering the transmission dynamics of COVID-19 in the population. In addition, given the limited data available and the analytic methods we used in the study, we could not directly estimate the effectiveness of some important interventions, such as face-mask wearing and personal hygiene practices, etc.

In conclusion, successful suppression of COVID-19 transmission in the first wave of the epidemic in Hong Kong was achieved through a combination of public health measures, focusing on early and near complete identification of cases and quickly interrupting chains of transmission as well as more general physical distancing measures in the community that stopped short of a society-wide lockdown. Serological studies are ongoing and are likely to indicate a very low level of population immunity following the first epidemic wave. Looking forward, broadened and intensive additional testing of high-risk sub-populations who have heightened susceptibility of infection and its complications as well as frequent and intense social mixing including pauci- and asymptomatic individuals, and targeted quarantine of arriving travellers from areas with ongoing COVID-19 transmission would reduce the risk of seeding a second wave in Hong Kong. Timely re-imposition of some or all of the public health measures listed above could be used to suppress future epidemic waves.

\section{Declarations}

\section{ACKNOWLEDGMENTS}

This project was supported by the Health and Medical Research Fund, Food and Health Bureau, Government of the Hong Kong Special Administrative Region (grant no. COVID190118), and the Theme-based Research Scheme (Project No. T11-712/19-N] of the Research Grants Council of the Hong Kong SAR Government.

\section{AUTHOR CONTRIBUTIONS}

All authors meet the ICMJE criteria for authorship. The study was conceived by PW, BJC and GML. Data analyses were done by PW, TKT, JYW, FH, HG, TWYN, DHC, DCA and EHYL. PW and BJC wrote the first draft of the manuscript, and all authors provided critical review and revision of the text and approved the final version.

\section{COMPETING INTERESTS STATEMENT}

BJC consults for Roche and Sanofi Pasteur. The authors report no other potential conflicts of interest.

\section{References}

1. Huang C, Wang Y, Li X, et al. Clinical features of patients infected with 2019 novel coronavirus in Wuhan, China. Lancet 2020; 395(10223): 497-506.

2. Li Q, Guan X, Wu P, et al. Early Transmission Dynamics in Wuhan, China, of Novel Coronavirus-Infected Pneumonia. N Engl J Med 2020; 382(13): 1199-207.

3. Zhu N, Zhang D, Wang W, et al. A Novel Coronavirus from Patients with Pneumonia in China, 2019. N Engl J Med 2020; 382(8): 727-33.

4. World Health Organization. Coronavirus disease (COVID-2019) situation reports. https://www.who.int/emergencies/diseases/novel-coronavirus-2019/situation-reports (accessed 11 May 2020.

5. Cohen J, Kupferschmidt K. Countries test tactics in 'war' against COVID-19. Science 2020; 367(6484): 1287-88.

6. Cowling BJ, Ali ST, Ng TWY, et al. Impact assessment of non-pharmaceutical interventions against coronavirus disease 2019 and influenza in Hong Kong: an observational study. Lancet Public Health 2020; 5(5): e279-e88.

Page $7 / 13$ 
7. Leung GM, Cowling BJ, Wu JT. From a Sprint to a Marathon in Hong Kong. N Engl J Med 2020; 382(18): e45.

8. Gandhi M, Yokoe DS, Havlir DV. Asymptomatic Transmission, the Achilles' Heel of Current Strategies to Control Covid-19. N Engl J Med 2020: doi: 10.1056/NEJMe2009758.

9. Lu X, Zhang L, Du H, et al. SARS-CoV-2 Infection in Children. N Engl J Med 2020; 382(17): 1663-5.

10. Giesecke J. Primary and index cases. Lancet 2014; 384(9959): 2024.

11. Leung GM, Quah S, Ho LM, et al. A tale of two cities: community psychobehavioral surveillance and related impact on outbreak control in Hong Kong and Singapore during the severe acute respiratory syndrome epidemic. Infect Control Hosp Epidemiol 2004; 25(12): 1033-41.

12. Leung GM, Ho LM, Chan SK, et al. Longitudinal assessment of community psychobehavioral responses during and after the 2003 outbreak of severe acute respiratory syndrome in Hong Kong. Clin Infect Dis 2005; 40(12): 1713-20.

13. Cowling BJ, $\mathrm{Ng} \mathrm{DM}$, Ip DK, et al. Community psychological and behavioral responses through the first wave of the 2009 influenza A(H1N1) pandemic in Hong Kong. J Infect Dis 2010; 202(6): 867-76.

14. Cori A, Ferguson NM, Fraser $C$, Cauchemez S. A new framework and software to estimate time-varying reproduction numbers during epidemics. Am J Epidemiol 2013; 178(9): 1505-12.

15. Fraser C. Estimating individual and household reproduction numbers in an emerging epidemic. PLoS One 2007; 2(8): e758.

16. Goldstein E, Dushoff J, Ma J, Plotkin JB, Earn DJ, Lipsitch M. Reconstructing influenza incidence by deconvolution of daily mortality time series. Proc Natl Acad Sci U S A 2009; 106(51): 21825-9.

17. He X, Lau EHY, Wu P, et al. Temporal dynamics in viral shedding and transmissibility of COVID-19. Nat Med 2020; 26: 672-5.

18. Salje H, Cummings DAT, Rodriguez-Barraquer I, et al. Reconstruction of antibody dynamics and infection histories to evaluate dengue risk. Nature 2018; 557(7707): 719-23.

19. Verity R, Okell LC, Dorigatti I, et al. Estimates of the severity of coronavirus disease 2019: a model-based analysis. Lancet Infect Dis 2020: doi: https://doi.org/10.1016/S473-3099(20)30243-7.

20. Hung IF, Lung KC, Tso EY, et al. Triple combination of interferon beta-1b, lopinavir-ritonavir, and ribavirin in the treatment of patients admitted to hospital with COVID-19: an open-label, randomised, phase 2 trial. Lancet 2020.

21. Cheng H, Jian S, Liu D, et al. Contact Tracing Assessment of COVID-19 Transmission Dynamics in Taiwan and Risk at Different Exposure Periods Before and After Symptom Onset. JAMA Intern Med 2020: doi: 10.1001/jamainternmed.2020.

22. Adam D, Wu P, Wong JY, et al. Clustering and superspreading potential of severe acute respiratory syndrome coronavirus 2 (SARS-CoV-2) infections in Hong Kong. Research Square 2020: doi: 0.21203/rs.3.rs-9548/v1.

23. Feng S, Shen C, Xia N, Song W, Fan M, Cowling BJ. Rational use of face masks in the COVID-19 pandemic. Lancet Respir Med 2020; 8(5): 434-6.

24. Greenhalgh T, Schmid MB, Czypionka T, Bassler D, Gruer L. Face masks for the public during the covid-19 crisis. BMJ 2020; 369: $\mathrm{m} 1435$.

25. Cheng VC, Wong SC, Chuang VW, et al. The role of community-wide wearing of face mask for control of coronavirus disease 2019 (COVID-19) epidemic due to SARS-CoV-2. J Infect 2020: doi: 10.1016/j.jinf.2020.04.024.

26. Cheng KK, Lam TH, Leung CC. Wearing face masks in the community during the COVID-19 pandemic: altruism and solidarity. Lancet 2020; S0140-6736(20): 30918-1.

\section{Table 1}

Table 1. Characteristics of COVID-19 cases confirmed in Hong Kong by case classification (see Appendix for definitions of sporadic imported, imported index, linked-imported, sporadic local, local index and linked-local cases). 


\begin{tabular}{|c|c|c|c|c|c|c|c|c|c|c|c|c|}
\hline & \multicolumn{2}{|c|}{$\begin{array}{c}\text { Sporadic } \\
\text { imported cases } \\
(n=680) \\
\end{array}$} & \multicolumn{2}{|c|}{$\begin{array}{c}\text { Imported index } \\
\text { cases } \\
(\mathrm{n}=32) \\
\end{array}$} & \multicolumn{2}{|c|}{$\begin{array}{c}\text { Linked-imported } \\
\text { cases } \\
(\mathrm{n}=67) \\
\end{array}$} & \multicolumn{2}{|c|}{$\begin{array}{l}\text { Sporadic local } \\
\text { cases }(n=46)\end{array}$} & \multicolumn{2}{|c|}{$\begin{array}{c}\text { Local index } \\
\text { cases }(n=22)\end{array}$} & \multicolumn{2}{|c|}{$\begin{array}{c}\text { Linked-local } \\
\text { cases }(n=198)\end{array}$} \\
\hline & Number & $(\%)$ & Number & (\%) & Number & (\%) & Number & $(\%)$ & Number & (\%) & Number & (\%) \\
\hline \multicolumn{13}{|l|}{ years } \\
\hline 15 & 20 & (3) & 0 & $(0)$ & 5 & (7) & 0 & $(0)$ & 0 & $(0)$ & 5 & (3) \\
\hline 14 & 466 & (69) & 15 & (47) & 26 & (39) & 27 & (59) & 7 & (32) & 123 & (62) \\
\hline j-64 & 156 & (23) & 14 & (44) & 25 & (37) & 15 & (33) & 9 & (41) & 51 & (26) \\
\hline \multirow[t]{2}{*}{; } & 38 & $(6)$ & 3 & (9) & 11 & (16) & 4 & (9) & 6 & (27) & 19 & (10) \\
\hline & 368 & (54) & 21 & (66) & 34 & (51) & 29 & (63) & 15 & (68) & 97 & (49) \\
\hline \multicolumn{13}{|l|}{$\begin{array}{l}\text { pation }^{1} \\
\text { estaurant/bar }\end{array}$} \\
\hline & 0 & $(0)$ & 0 & $(0)$ & 1 & (10) & 0 & $(0)$ & 0 & $(0)$ & 42 & (61) \\
\hline \multicolumn{13}{|l|}{ irport/airline } \\
\hline \multicolumn{13}{|l|}{ ealth care } \\
\hline ers & 2 & (1) & 0 & (0) & 0 & $(0)$ & 1 & (7) & 1 & (25) & 3 & $(4)$ \\
\hline \multicolumn{13}{|l|}{ r/private } \\
\hline r & 11 & $(4)$ & 1 & (50) & 4 & $(40)$ & 1 & (7) & 0 & & 4 & (6) \\
\hline зacher & 3 & (1) & 0 & $(0)$ & 1 & (10) & 2 & (13) & 0 & $(0)$ & 0 & $(0)$ \\
\hline /taxi driver & 1 & $(0.4)$ & 0 & (0) & 0 & $(0)$ & 2 & (13) & 1 & (25) & 1 & (1) \\
\hline \multicolumn{13}{|l|}{ dresser/beauty } \\
\hline ur employee & 0 & $(0)$ & 0 & $(0)$ & 0 & $(0)$ & 2 & (13) & 0 & $(0)$ & 3 & $(4)$ \\
\hline n employee & 0 & (0) & 0 & (0) & 0 & (0) & 0 & $(0)$ & 0 & (0) & 1 & (1) \\
\hline slice & 0 & (0) & 0 & (0) & 0 & $(0)$ & 2 & (13) & 0 & $(0)$ & 2 & (3) \\
\hline urity guard & 0 & $(0)$ & 0 & (0) & 0 & $(0)$ & 0 & $(0)$ & 0 & $(0)$ & 3 & (4) \\
\hline sur guide & 1 & $(0.4)$ & 0 & (0) & 0 & (0) & 0 & $(0)$ & 0 & $(0)$ & 0 & $(0)$ \\
\hline $\mathrm{ers}^{2}$ & 251 & (88) & 0 & (0) & 2 & (20) & 3 & (20) & 1 & (25) & 5 & (7) \\
\hline \multicolumn{13}{|l|}{ orbidities } \\
\hline iabetes & 16 & (2) & 3 & (9) & 2 & (3) & 3 & (7) & 4 & (18) & 9 & (5) \\
\hline ıertension & 20 & (3) & 2 & (6) & 3 & (4) & 2 & (4) & 4 & (18) & 14 & (7) \\
\hline besity & 2 & $(0.3)$ & 0 & (0) & 0 & (0) & 0 & (0) & 1 & (5) & 2 & (1) \\
\hline ignant & & & & $(0)$ & & & & $(0)$ & & (0) & & (0) \\
\hline lasms & 3 & $(0.4)$ & 0 & & 2 & (3) & 0 & & 0 & & 0 & \\
\hline hronic liver & & $(0.4)$ & & $(0)$ & & & & $(0)$ & & $(0)$ & & $(0)$ \\
\hline ses & 3 & & 0 & & 1 & (1) & 0 & & 0 & & 0 & \\
\hline ney diseases & 3 & $(0.4)$ & 2 & (6) & 0 & $(0)$ & 1 & (2) & 2 & (9) & 2 & (1) \\
\hline \multicolumn{13}{|l|}{ ist reported $\mathrm{O}_{2}$} \\
\hline \multicolumn{13}{|l|}{ ation $^{3}$} \\
\hline $75 \%$ & 17 & $(47)$ & 1 & (33) & 6 & (67) & 2 & (29) & 2 & (25) & 3 & (19) \\
\hline o to $89 \%$ & 6 & (17) & 0 & $(0)$ & 2 & (22) & 1 & (14) & 0 & $(0)$ & 5 & (31) \\
\hline )\% to $94 \%$ & 6 & (17) & 2 & (67) & 0 & $(0)$ & 1 & (14) & 4 & (50) & 6 & (38) \\
\hline$\%$ & 7 & (19) & 0 & $(0)$ & 1 & (11) & 3 & (43) & 2 & (25) & 2 & (12) \\
\hline \multicolumn{13}{|l|}{ reported $\mathrm{O}_{2}$} \\
\hline ation < $<95 \%$ & 29 & (4) & 3 & (9) & 8 & (12) & 4 & (9) & 6 & (27) & 14 & (7) \\
\hline \multirow{3}{*}{$\begin{array}{l}\text { y distribution } \\
\text { nset to } \\
\text { italization, }\end{array}$} & Mean & (sd) & Mean & (sd) & Mean & (sd) & Mean & (sd) & Mean & (sd) & Mean & (sd) \\
\hline & & & & & & & & & & & & \\
\hline & 6.4 & $(6.1)$ & 4.2 & (3.1) & 4.4 & $(4.4)$ & 6.2 & $(2.8)$ & 5.3 & $(4.0)$ & 4.5 & $(4.1)$ \\
\hline iet to & & & & & & & & & & & & \\
\hline $\begin{array}{l}\text { rmation, days } \\
\text { nset to }\end{array}$ & 7.1 & $(6.1)$ & 5.0 & $(3.2)$ & 5.5 & $(4.5)$ & 6.8 & (2.8) & 6.2 & $(4.1)$ & 5.3 & $(4.0)$ \\
\hline targe, days & 27.3 & (10.8) & 26.6 & $(9.8)$ & 29.2 & (13.2) & 25.4 & $(9.5)$ & 35.6 & $(17.1)$ & 28.6 & (10.9) \\
\hline & 17.0 & NA & 6.0 & NA & NA & & NA & & 9.0 & NA & 17.0 & NA \\
\hline firmation to last & & & & & & & & & & & & \\
\hline ive sample, days & 15.6 & $(11.3)$ & 17.3 & $(11.0)$ & 15.0 & $(10.5)$ & 13.8 & $(10.5)$ & 21.0 & $(15.1)$ & 19.0 & $(11.7)$ \\
\hline
\end{tabular}


NA: not available

1284 (42\%) of sporadic imported cases, 2 (6\%) of imported index cases, 10 (15\%) of linked-imported cases, 15 (33\%) sporadic local cases, 4 (18\%) of local index cases, and 69 (35\%) of linked-local cases reported their occupation.

2 Others include supervisor, clerk, call centre staff, factory worker, overseas student, and reporter.

336 (5\%) of sporadic imported cases, 3 (9\%) of imported index cases, 9 (13\%) of linked-imported cases, 7 (15\%) of sporadic local cases, 8 (36\%) of local index cases, and 16 (8\%) of linked-local cases monitored for $\mathrm{O}_{2}$ saturation.

\section{Figures}

A

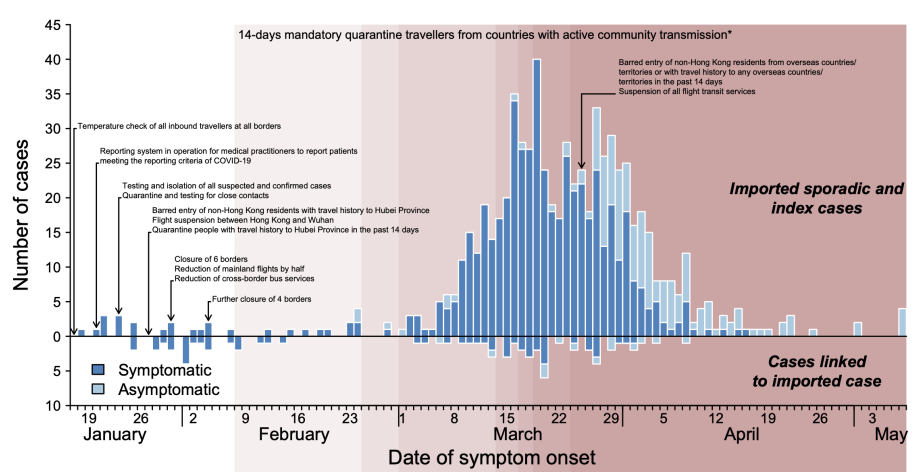

B

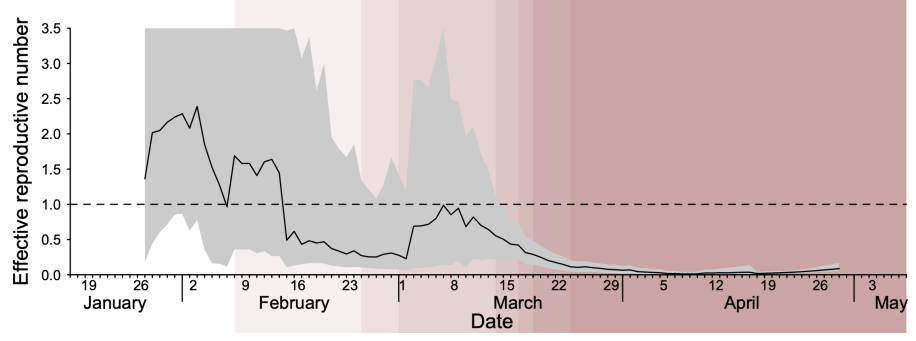

c

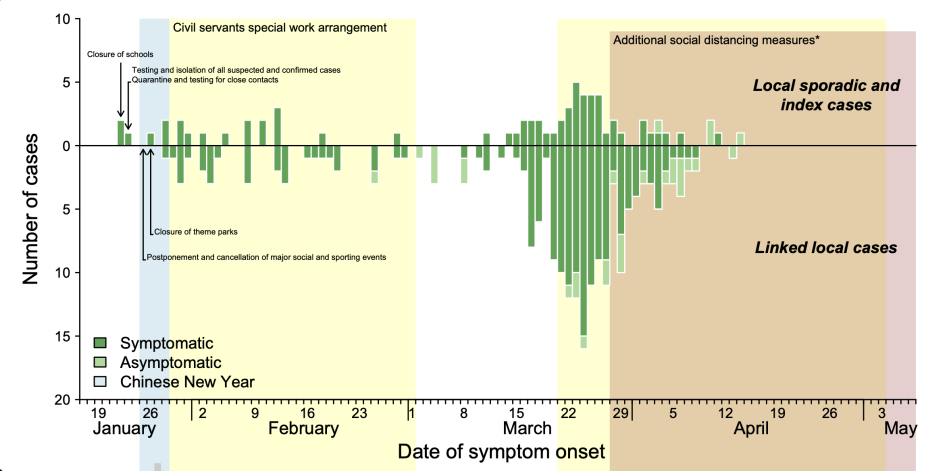

D

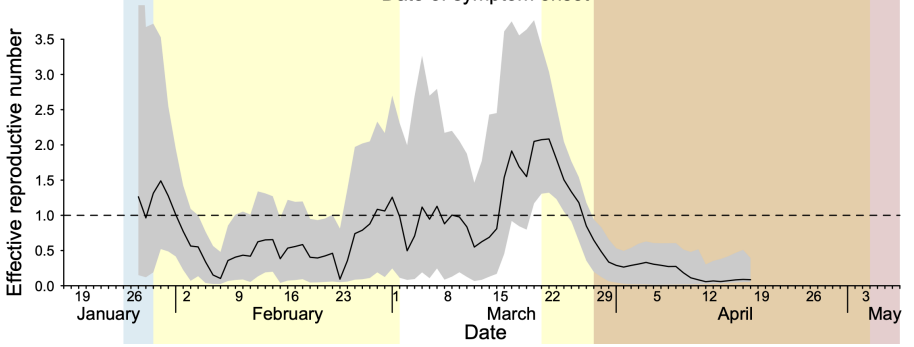

\section{Figure 1}

Panel A: Sporadic imported COVID-19 cases and index cases linked to imported COVID-19 cases (above), and other cases linked to imported cases (below), by date of symptom onset for symptomatic infections and by date of confirmation for asymptomatic infections. Panel B: Estimated effective reproduction numbers (Rt) for infections of imported cases in Hong Kong. The shaded areas in red indicate the implementation of 14-day mandatory quarantine for all inbound travellers from places with active community transmission of COVID-19 (details in Appendix), with darker colours showing the expansion of quarantine order targeting travellers from wider geographic areas. Panel C: Local sporadic cases and index cases linked to local cases (above), and other cases linked to local cases (below) by date of symptom onset for symptomatic infections and by date of confirmation for asymptomatic infections. Panel D: Estimated Rt for local transmission based on identified local cases. Two yellow shaded areas show the time periods adopting the work from home policy for civil servants in Hong Kong. The pink area indicates the implementation of additional physical distancing measures (details in Appendix, Community measures). The blue bar indicates the Lunar New Year holidays. Shaded 

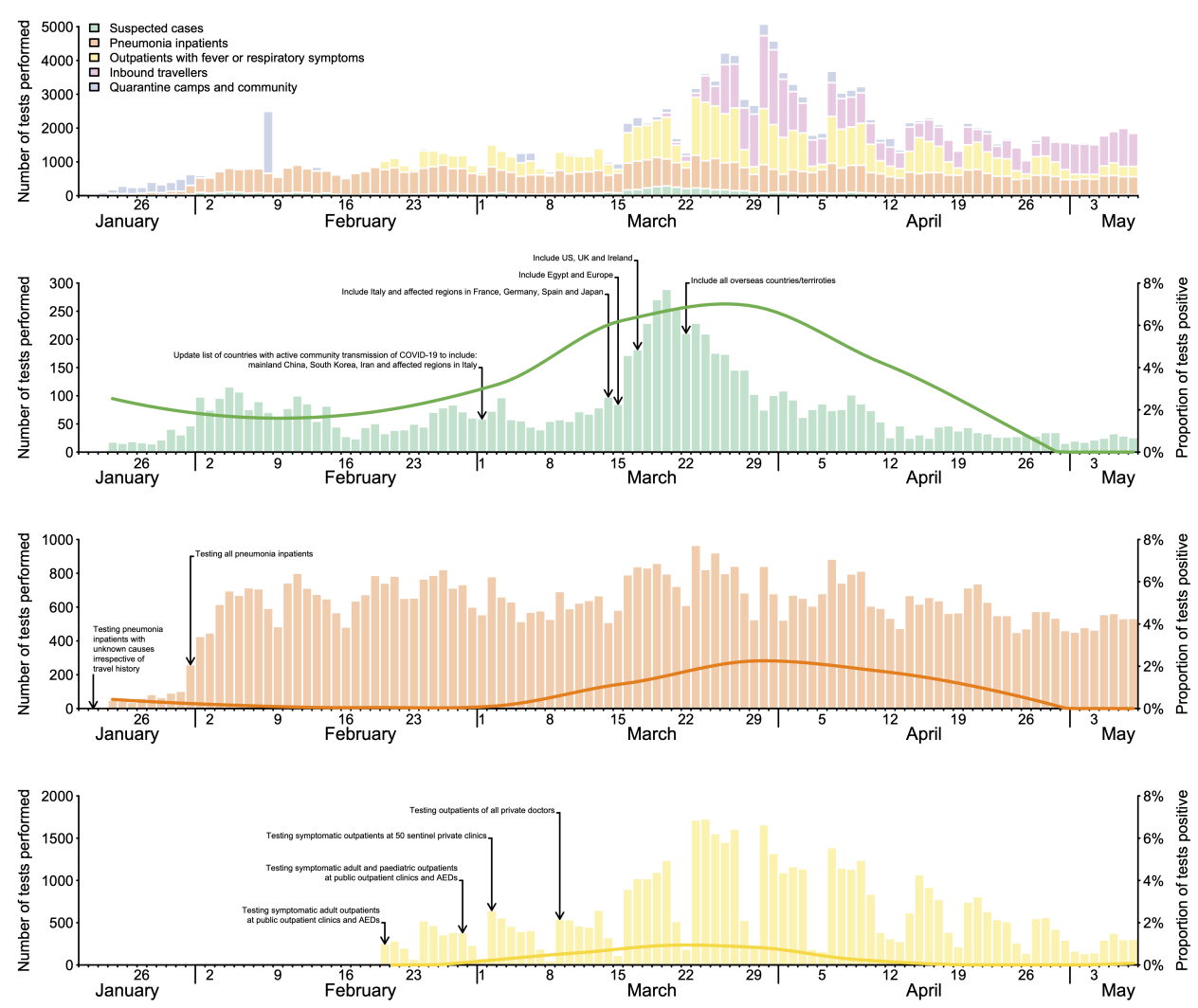

E
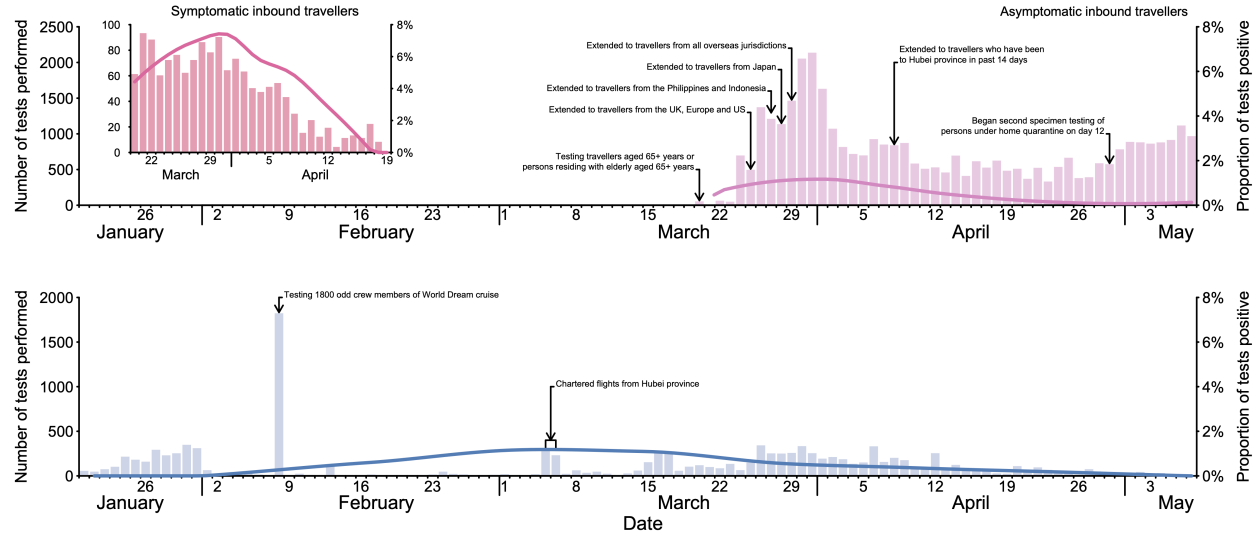

Figure 2

Daily laboratory testing frequencies (bars) and COVID-19 case detection proportions (lines) in Hong Kong, A) by source of specimen collection, B) among individuals who were suspected COVID-19 cases (meeting the reporting criteria of COVID-19) (green), C) among inpatients with pneumonia admitted into hospital (orange), D) among outpatients presenting fever or respiratory symptoms (blue), E) among asymptomatic (light pink) and symptomatic (pink) inbound travellers, and F) in specimens received from public hospitals or private healthcare sectors, stool specimens, environmental specimens, and from persons in quarantine camps or under medical observation/surveillance (blue). Screening tests have been also carried out in Hong Kong from 24 April in people who were perceived to have a potentially high risk of infection. During 24 April - 7 May, in total 127 specimens were collected (< 20 specimens per day) from immigration offenders who were newly admitted into the Castle Peak Bay Immigration for detention and awaiting repatriation/removal/deportation, and no specimens were tested positive for SARS-CoV-2 (data not shown in this figure). 

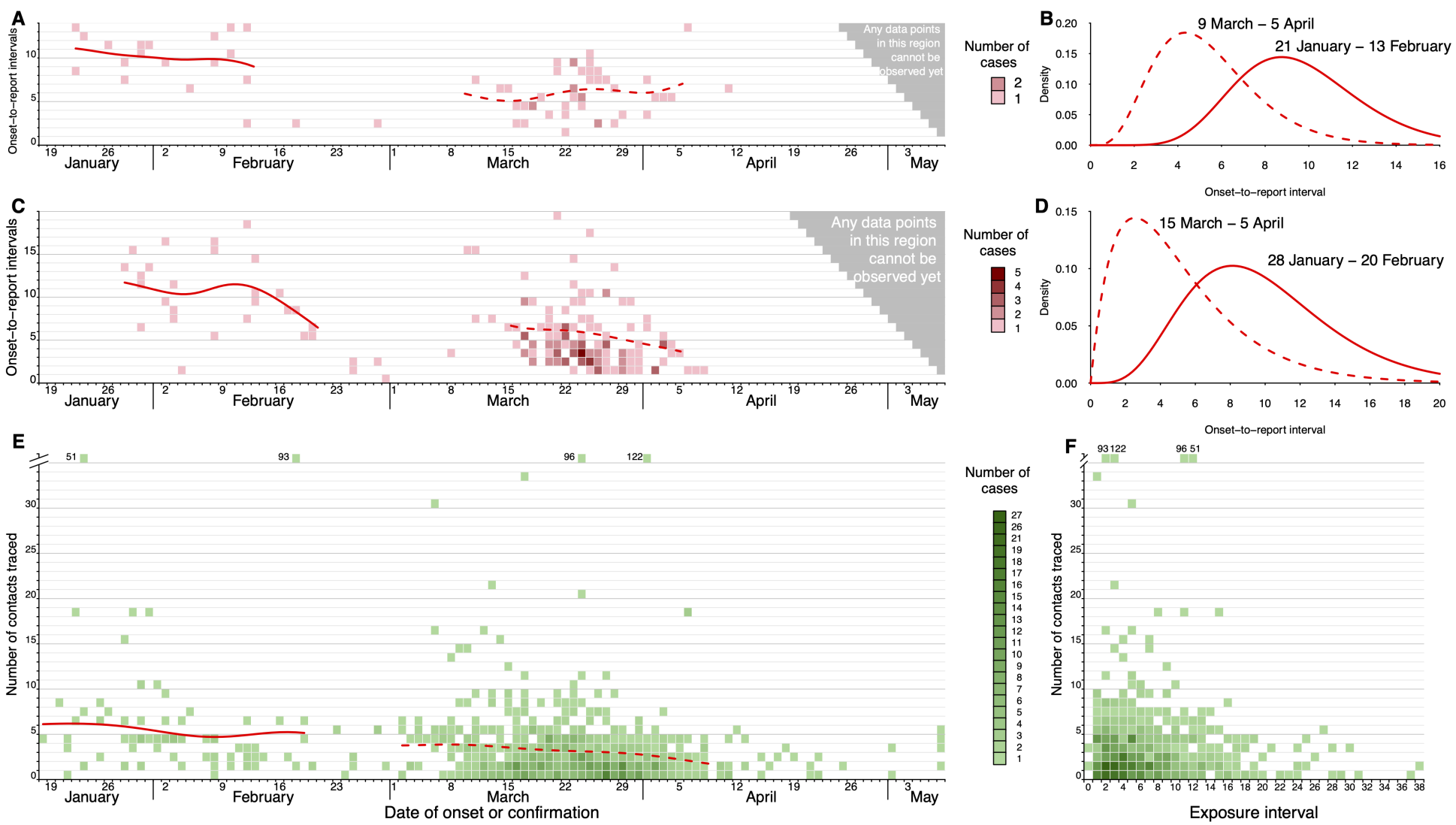

\section{Figure 3}

Time delays in identification of sporadic local cases and index cases linked to local cases (Panels A and B) and delays in confirming cases who were contacts of local cases (Panels C and D) in Hong Kong during January-February (early phase) and March-April (late phase) in the first wave of the COVID-19 epidemic in Hong Kong. Panel E: Reported numbers of close contacts (squares) and the estimated average number of close contacts (lines) by onset date (for symptomatic cases) or confirmation date (for asymptomatic cases) for imported and locally infected cases. Panel F: Reported numbers of close contacts of imported and locally infected cases against the time delay of the confirmed cases from onset (for symptomatic cases) or last exposure (for asymptomatic cases) to laboratory confirmation. Three sporadic asymptomatic local cases were excluded in Panel E and Panel $\mathrm{F}$ due to the lack of information on the time of their last exposure to a confirmed case. 


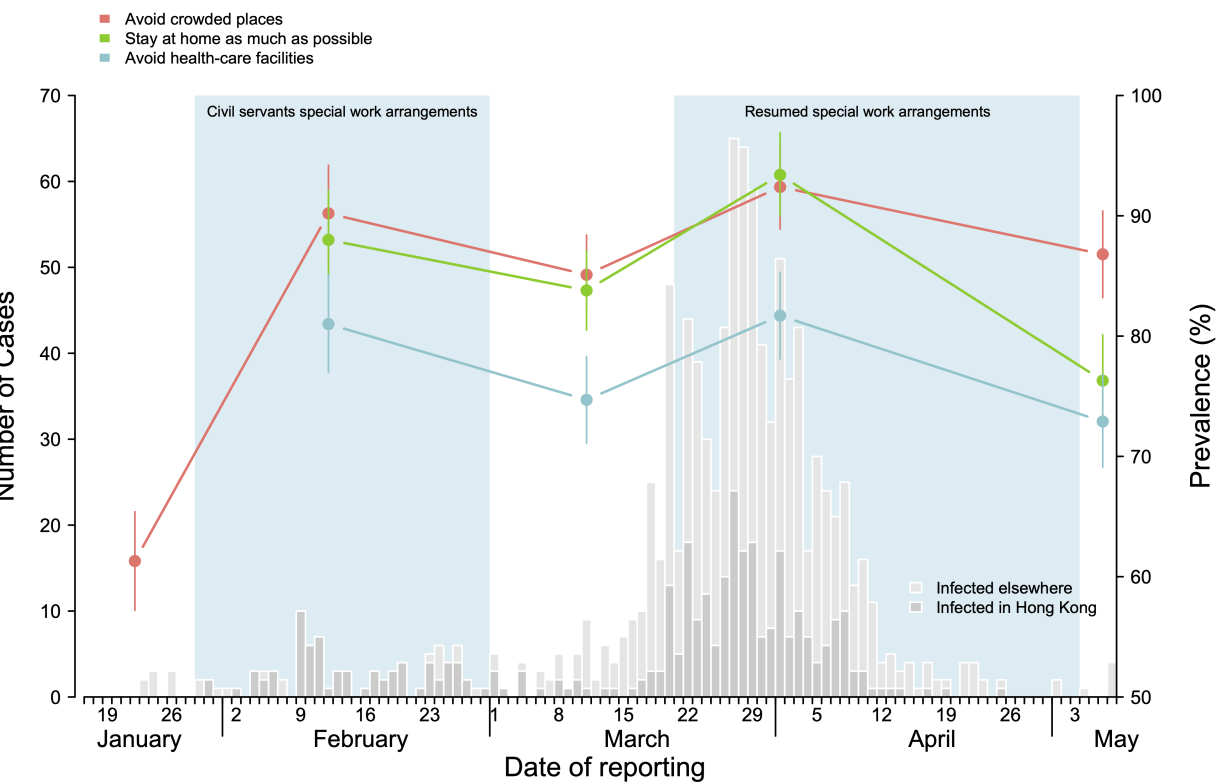

B

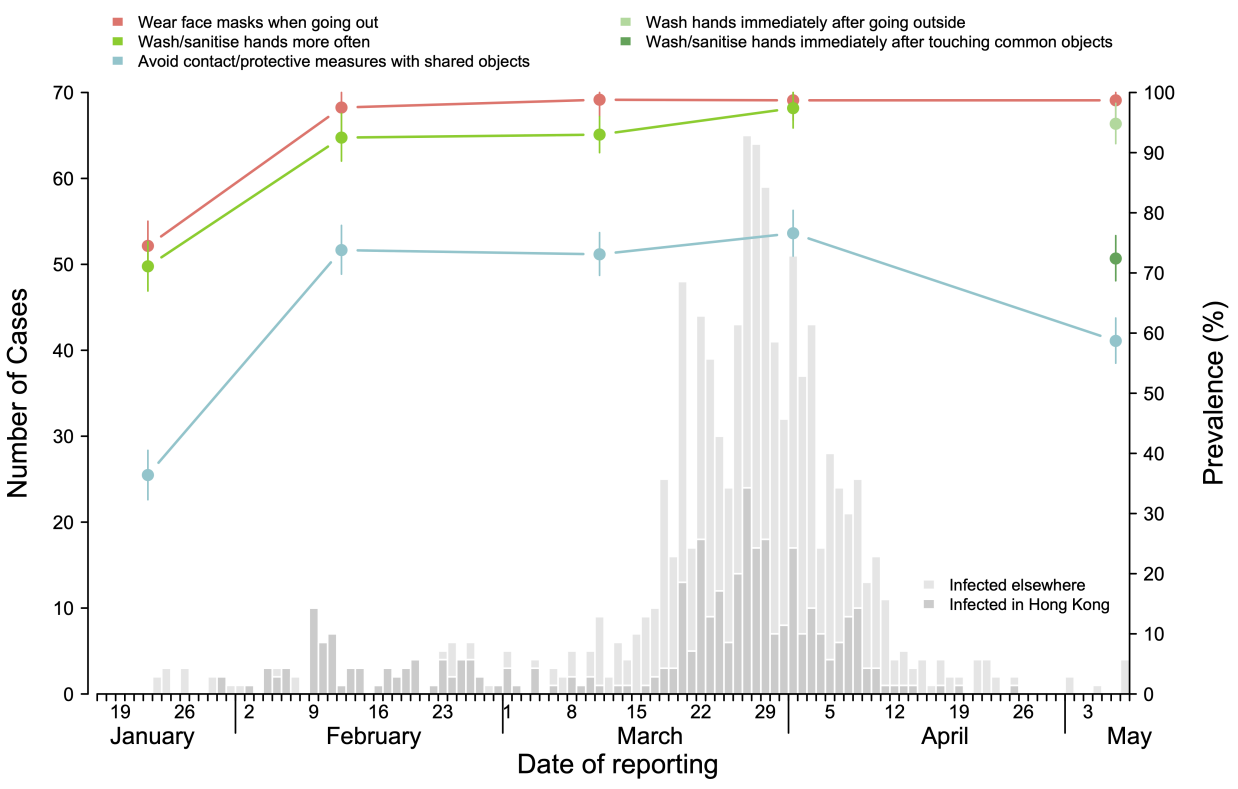

Figure 4

Population behaviours related to physical distancing (Panel A) and personal hygiene (Panel B) measured in participants responding to the five cross-sectional telephone surveys conducted among the general adult population in Hong Kong from January to April 2020. Bars show the daily numbers of COVID-19 cases identified in Hong Kong by reporting date, with cases infected in Hong Kong in darker grey and cases infected elsewhere in lighter grey.

\section{Supplementary Files}

This is a list of supplementary files associated with this preprint. Click to download.

- Appendix.docx 